\title{
Compression of Multilayered Composite Electrospun Scaffolds: A Novel Strategy to Rapidly Enhance Mechanical Properties and Three Dimensionality of Bone Scaffolds
}

\author{
Parthasarathy A. Madurantakam, ${ }^{1,2}$ Isaac A. Rodriguez, ${ }^{1}$ Koyal Garg, ${ }^{1}$ Jennifer M. McCool, \\ Peter C. Moon, ${ }^{3}$ and Gary L. Bowlin ${ }^{1,4}$ \\ ${ }^{1}$ Tissue Engineering Laboratory, Department of Biomedical Engineering, Virginia Commonwealth University, \\ P.O. Box 843067, Richmond, VA 23284-3067, USA \\ ${ }^{2}$ School of Dentistry, Virginia Commonwealth University, P.O. Box 843067, Richmond, VA 23284-3067, USA \\ ${ }^{3}$ Biomaterials Laboratory, School of Dentistry, Virginia Commonwealth University, P.O. Box 843067, Richmond, VA 23284-3067, USA \\ ${ }^{4}$ Department of Biomedical Engineering, School of Engineering, Virginia Commonwealth University, P.O. Box 843067, Richmond, VA \\ 23284-3067, USA
}

Correspondence should be addressed to Gary L. Bowlin; glbowlin@vcu.edu

Received 6 July 2012; Accepted 14 December 2012

Academic Editor: Manish U. Chhowalla

Copyright (C) 2013 Parthasarathy A. Madurantakam et al. This is an open access article distributed under the Creative Commons Attribution License, which permits unrestricted use, distribution, and reproduction in any medium, provided the original work is properly cited.

\begin{abstract}
One major limitation of electrospun scaffolds intended for bone tissue engineering is their inferior mechanical properties. The present study introduces a novel strategy to engineer stiffer scaffolds by stacking multiple layers and cold welding them under high pressure. Electrospun polydioxanone (PDO) and PDO:nanohydroxyapatite (PDO:nHA) scaffolds (1, 2, or 4 layered stacks) were compressed either before or after mineralizing treatment with simulated body fluid (SBF). After two weeks in SBF, scaffolds were analyzed for total mineral content and stiffness by Alizarin red S and uniaxial tensile testing, respectively. Scaffolds were also analyzed for permeability, pore size, and fiber diameter. Results indicated that compression of multiple layers significantly increased the stiffness of scaffolds while reducing mineralization and permeability. This phenomenon was attributed to increased density of fibers and loss of surface area due to fiber welding. Statistics revealed, the 4-layered PDO:nHA scaffold compressed first followed by mineralization in revised SBF had maximal stiffness, low permeability and pore size, and mineralization second only to noncompressed scaffolds. Within the limitations of permeability and pore size, this scaffold configuration represents an optimal midway for desired stiffness and mineral content for bone tissue engineering.
\end{abstract}

\section{Introduction}

Tissue engineering and artificial organs face a twin challenge of satisfying the mechanical and biological properties of the tissues they intend to replace. The skeletal system, comprising of bones, play a vital role in load bearing, protecting internal organs and calcium homeostasis [1]. Even though adult bones retain a significant capacity to regenerate following injury, this healing response is limited by the size of the defect, often referred to as critical sized defect. When bone defects exceed this critical size (varies with the species), external therapeutic intervention becomes necessary to restore original function.
This finding has driven research to develop optimal bone substitutes that can be grafted in the defect site. Among the foremost requisites for biomaterials to be mechanically and biologically adequate are its stiffness, ability to integrate with native bone and undergo physiological bone remodeling [2$4]$.

Traditional replacements include metals, ceramics, and polymers having excellent bulk material properties that are often superior to native bone in stiffness. Since the primary function of bone was identified to be mechanical, early efforts were to engineer a biomaterial as strong and stiff as possible. As a result, metals, ceramics, and polymers were developed 
with excellent bulk material properties that often exceeded the stiffness of native bone. After witnessing some initial success, many implants failed because of a phenomenon called stress shielding. In this process, load bearing is primarily taken up by the implant and the surrounding bone is protected from the same stresses. After being chronically exposed to low loads (due to ineffective transfer of stresses), the native bone cells respond by bone resorption around the implant, eventually leading to its failure. Now, efforts are made to engineer synthetic bone substitutes that match the native bone in its physical properties. In addition to matching the stiffness, achieving bonding of the implant to the native bone is another strategy to ensure effective stress transfer. The development of bio-glass that can chemically bond to bone is an important advance in this regard $[5,6]$.

Excluding certain ceramics, conventional bone replacements being biologically inert cannot induce a specific cellular interaction. They are not susceptible to physiological bone remodeling induced by changing load patterns, parathyroid hormone, and calcium homeostasis. In this regard, the next challenge is the development of true bone substitutes that replace all of the mechanical and biological functions. Tissue engineering is an attractive strategy to the development of synthetic bone substitutes because the implant is present for a finite period of time during which it should induce formation of native bone. In this circumstance, the long-term issues of remodeling and load transfer become irrelevant. The challenge of bone tissue engineering is to develop a scaffold that matches the healing bone in its mechanical properties and yet reliably induce new bone formation in the most efficient manner [5, 7-9].

Electrospinning is a versatile fabrication strategy that lends itself well to the field of tissue engineering because it replicates the native extracellular matrix (ECM) in terms of composition and nanofibrillar architecture. Even though electrospinning offers great promise in generating biologically optimal scaffolds with respect to its geometry and dimensions, the fact that these scaffolds are more than $90 \%$ porous makes them inferior in their mechanical properties. Since cell migration and implant integration hinges on the porosity of electrospun scaffolds, effort to improve their physical properties without compromising this important property is a pertinent area of research [10-12].

Bone is a structural composite with brittle, inorganic hydroxyapatite filler particles closely associated with a tough organic collagenous matrix. Given the role of hydroxyapatite in determining mechanical properties, various approaches have been used to incorporate nanocrystalline hydroxyapatite (nHA) into electrospun scaffolds [13-17]. In previous studies conducted in our laboratory, we demonstrated our ability to successfully load nHA within scaffolds and subsequently mineralize them by treatment in simulated body fluid $(\mathrm{SBF})$. The results of this previous study revealed that the electrospun polydioxanone (PDO) scaffolds containing $50 \%$ nHA (PDO:nHA) incubated in $1 \mathrm{x}$ revised SBF ( $\mathrm{r}-$ $\mathrm{SBF}$ ) resulted in maximum mineralization. In the present study, we introduce cold-welding as a novel yet simple strategy to significantly improve the mechanical properties of electrospun scaffolds, specifically PDO and PDO:nHA scaffolds.

\section{Materials and Methods}

2.1. Scaffold Preparation by Electrospinning. Polydioxanone (PDO, Ethicon, Inc., NJ, USA) and nanocrystalline hydroxyapatite (nHA, Berkeley Advanced Biomaterials Inc, San Leandro, CA, USA) were used to generate two types of scaffolds: PDO and PDO:nHA composite scaffolds. PDO was dissolved at $100 \mathrm{mg} / \mathrm{mL}$ concentration in electrospinning solvent, 1, 1, 1, 3, 3, 3 hexafluoro-2-propanol (HFP) to generate pure PDO scaffolds. nHA (50\% by wt. to PDO) was dispersed in HFP by ultrasonication for 10 minutes on pulse mode (on: $50 \mathrm{~s}$, off: $10 \mathrm{~s}$ ) at $38 \%$ maximum amplitude using a Cole-Palmer Ultrasonic Processor sonicator (model $\mathrm{CPX}$ ) prior to addition of PDO to generate composite scaffolds. Electrospinning conditions were optimized (needle size: 16 gauge, flow rate: $7 \mathrm{~mL} /$ hour, air-gap distance: $20 \mathrm{~cm}$, voltage: $22 \mathrm{kV}$ ) to generate continuous nonwoven composite nanofibers that were collected onto a rotating rectangular mandrel $(7.5 \times 2.5 \times 0.5 \mathrm{~cm})$. After electrospinning, scaffolds were removed from the mandrel, dried in a fume hood for $30 \mathrm{~min}$, and cut to form $2.3 \times 7.3 \mathrm{~cm}$ sheets with an average thickness of $207.4 \mu \mathrm{m}$ and $317.5 \mu \mathrm{m}$ for PDO and PDO:nHA, respectively.

2.2. Scanning Electron Microscopy. For scanning electron microscopy, air-dried electrospun scaffolds were mounted on aluminum stubs, sputter coated with gold, and examined at an accelerating voltage of $10 \mathrm{kV}$ using Zeiss EVO 50 XVP scanning electron microscope. Fiber diameters $(n=60)$ were calculated from the SEM images using UTHSCSA Image Tool 3.0 software.

2.3. Scaffold Compression. All scaffolds were cut into flat rectangular sheets carefully avoiding any raised edges. The sheets were then placed flush on top of each other in between two flat stainless steel blocks. This assembly was compressed to 20,000 psi using a mechanical hydraulic press (Carver, Inc. Wabash, IN, USA) and the pressure held for one minute. At this time, the pressure was released, and scaffolds were removed and stored in a desiccator for either SBF treatment or mechanical testing. The number of layers of flat sheets compressed using the technique described was 1,2 , or 4 . We also tested a single layer uncompressed electrospun scaffold of identical dimensions for control. The 2 or 4 multilayered scaffolds were either mineralized as individual sheets first followed by their compression (designated C2M1) or compressed first and mineralized as a unit (designated C1M2).

2.4. Biomimetic Mineralization with Simulated Body Fluid. Revised simulated body fluid ( $\mathrm{r}-\mathrm{SBF}$ ) was prepared following a published protocol [18]. The cut flat rectangular electrospun sheets were incubated in $20 \mathrm{~mL} \mathrm{r}$-SBF in a $100 \mathrm{~mm} \times 15 \mathrm{~mm}$ Petri dish at $37^{\circ} \mathrm{C}$ in $5 \% \mathrm{CO}_{2}$ atmosphere for 14 days with SBF solutions renewed every 5 days. 
2.5. Quantification of Mineralization Using Alizarin Red $S$ (ARS). A stock solution of $40 \mathrm{mM}$ Alizarin red S (ARS, Sigma, MO, USA) pH 4.1 was prepared in deionized (DI) water and quantification of mineralization was achieved by following a modified published protocol $[19,20]$. After incubation in $1 \mathrm{x} \mathrm{r}$-SBF for 14 days, $10 \mathrm{~mm}$ discs were punched ( $n=6)$ from the mineralized sheets, rinsed in DI water, fixed in $3.7 \%$ buffered formaldehyde for $30 \mathrm{~min}$, and stained with an excess of ARS solution for $20 \mathrm{~min}$. After the unbound dye was washed off, the bound stain was solubilized in $50 \%$ acetic acid. After $30 \mathrm{~min}$, the solubilized stain was pipetted out, $\mathrm{pH}$ adjusted to 4.1, and absorbance measured at 550 $\mathrm{nm}$ in a 96-well plate using SpectraMax spectrophotometer (Molecular Devices). Control scaffolds were incubated under similar conditions in DI water.

2.6. Mechanical Testing. The scaffolds $(n=6)$ were punched into dog bones with a metal punch $(2.75 \mathrm{~mm}$ wide at their narrowest point, gage length of $7.5 \mathrm{~mm}$ ) and individual specimen thickness was measured on a Mitutoyo IP54 digital micrometer (Mitutoyo American Corp.). The dog bones were subjected to uniaxial tensile testing using a MTS Bionix 200 testing system ( $50 \mathrm{~N}$ load cell) with an extension rate of $10 \mathrm{~mm} / \mathrm{min}$. Elastic modulus, strain at break and energy to break were recorded by the MTS software TestWorks 4.0.

2.7. Permeability and Pore Size Measurements. Both types of scaffolds-C1M2 and C2M1 containing 1, 2, or 4 layers, were subjected to permeability and pore size measurements following the protocol developed by Sell et al. [21]. Briefly, a graduated $10 \mathrm{~mL}$ pipette was placed horizontally that was connected to a reservoir at a height of $150 \mathrm{~cm}$ from the scaffold. The electrospun scaffold was compressed between two silicone gaskets and a large-pore metal screen was placed on the underside of the scaffold to prevent excessive distension of the test scaffold. Fluid flow was recorded every minute for the first $10 \mathrm{~min}$ of testing and the permeability was mathematically calculated using Darcy's equation. These permeability values were then used to determine the average pore sizes of the electrospun scaffold, again using the derived equation.

2.8. Statistical Analysis. All statistical analysis of the data was based on a Kruskal-Wallis one-way analysis of variance on ranks and a Tukey-Kramer pairwise multiple comparison procedure $(\alpha=0.05)$ performed with JMP IN 7 statistical software (SAS Institute).

\section{Results}

3.1. Integrity of Multilayered Scaffolds. A potential concern in the development of multilayered scaffolds was the delamination of individual layers. In the experiments conducted, this problem was not encountered. The scaffolds, irrespective of the composition (PDO or PDO:nHA), compression sequence (C1M2 or C2M1), or number of layers (2 or 4 ) after compression held together surprisingly well even during the mechanical testing. It was only when the number of layers increased to 8 that delamination occurred. Hence, we limited our studies to 4 layers. It is our hypothesis that high pressure generated by the hydraulic press induces attractive intermolecular forces among the polymeric molecules within the nanofibers and fusion of adjacent layers. The cumulative effect of these dominant attractive noncovalent, van der Waals' forces result in welding/bonding of the fibers at the nanoscale. More interestingly, this effect was predominant in the composite PDO:nHA scaffolds. Distinct areas of deformation and compression were readily observed in these scaffolds compared to the pure PDO scaffolds. This observation could be partly explained by the fact that $\mathrm{PDO}$ is an elastic polymer and resisted plastic deformation during compression even at a nanoscale. Incorporation of $\mathrm{nHA}$ during the composite scaffold fabrication rendered the scaffolds more prone to plastic deformation and presumably forced the nanofibers close enough to cause new bonds to form.

3.2. Scanning Electron Microscopy. There are two striking observations from the SEM in Figure 1: (a) the morphology of PDO scaffolds is entirely different from that of PDO:nHA composite scaffolds; (b) within the PDO:nHA scaffolds there is a remarkable difference in the morphology and architecture depending on which protocol was used for compression C1M2 or C2M1. The occasional fiber fracture seen in the SEM is a natural consequence of incubation in $\mathrm{SBF}$ and has been reported in the previous study as well [19]. PDO scaffolds showed no significant changes in the architecture, irrespective of the number of layers or compression sequence. Alternatively, electrospun PDO:nHA scaffolds exhibited interesting behavior: there is a distinct difference in the scaffold morphology depending on whether the layers were compressed first then mineralized (C1M2) or mineralized first and compressed after (C2M1). Scaffolds compressed first (C1M2) showed discrete flattened out areas, presumably indicating areas of contact between adjacent layers during compression. Such intimate contact of fibers at very high pressure may have induced binding between adjacent layers. None of the multilayered scaffolds showed any signs of spontaneous delamination. This cumulative effect of multiple fibers welding among different layers neatly correlate to an increased stiffness observed in scaffolds subjected to compression.

The analysis of mean fiber diameter (Figure 2) for each condition showed that PDO:nHA scaffolds had smaller fibers than PDO scaffolds. Furthermore PDO:nHA scaffolds had a more uniform fiber size while PDO scaffolds contained a variety of small and large fibers. Overall the fiber diameters for each scaffold type were not affected by compression or mineralization.

3.3. Quantification of Mineralization by ARS. Statistical analysis shows superior performance of PDO:nHA scaffolds compared to PDO scaffolds for all conditions. In addition, there is no statistical difference between the number of layers with PDO scaffolds compared to the order of compression and mineralization. Similarly, mineralization of PDO:nHA 


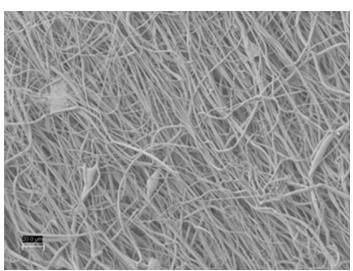

(a)

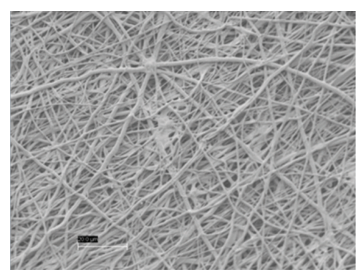

(e)

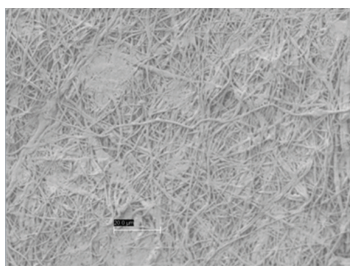

(i)

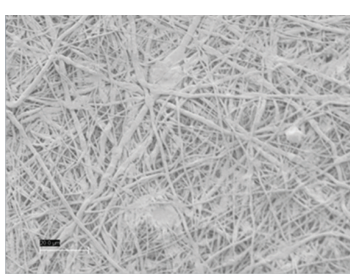

$(\mathrm{m})$

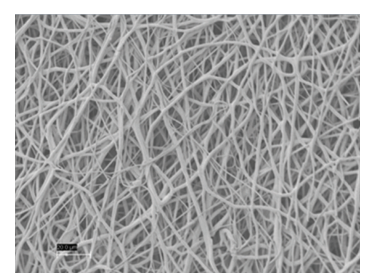

(b)

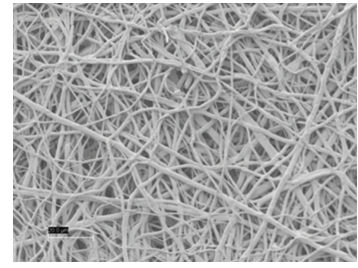

(f)

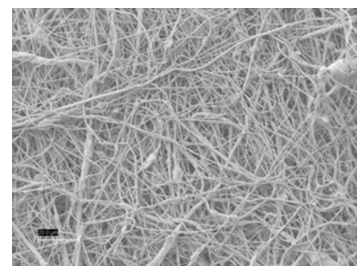

(j)

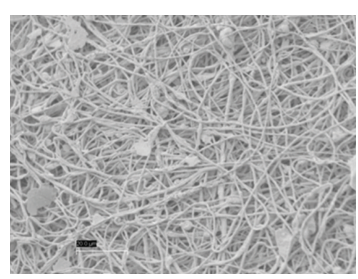

(n)

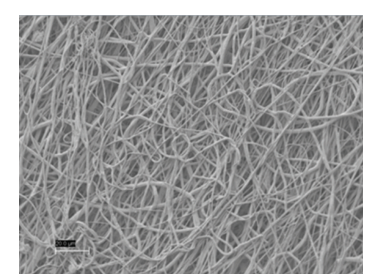

(c)

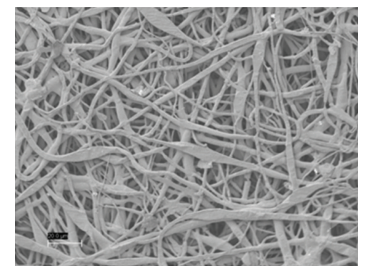

(g)

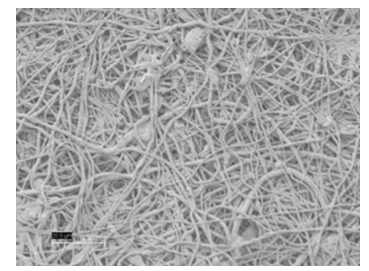

(k)

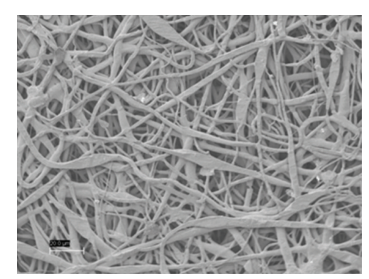

(o)

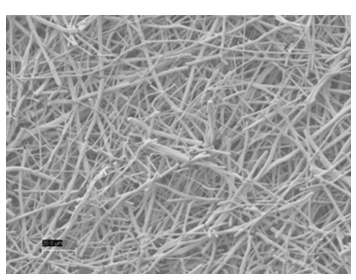

(d)

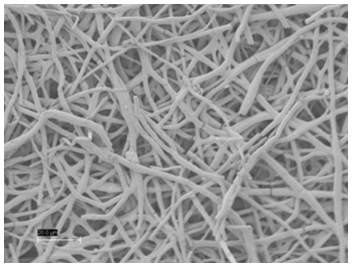

(h)

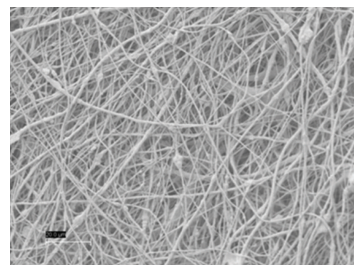

(1)

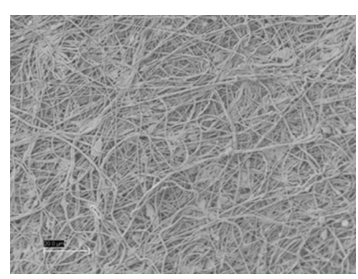

(p)

FIGURE 1: SEM of electrospun PDO scaffolds after biomimetic mineralization in SBF for 2 weeks. (a)-(b) Compressed and noncompressed control scaffolds in deionized water. (c)-(d) 1-layer compressed and noncompressed scaffolds in SBF, (e)-(f) 2-layers C1M2 and C2M1, (g)-(h) 4-layers C1M2 and C2M1. PDO scaffolds containing 50\% nHA are designated in the same format. (i)-(j) Compressed and noncompressed control scaffolds in deionized water. (k)-(l) 1 layer compressed and noncompressed scaffolds in SBF, (m)-(n) 2 layers C1M2 and C2M1, and (o)-(p) 4-layers C1M2 and C2M1. Scale bars $=20 \mu \mathrm{m}$.

scaffolds is independent of multiple layers. Interestingly, PDO:nHA scaffolds mineralize differently depending on whether compression was done before or after mineralization. Compression of PDO:nHA scaffolds before SBF treatment is identical for 1- and 2-layered scaffolds, while the 4-layered scaffolds had superior mineralization. However, the strategy of mineralizing multiple scaffolds separately followed by compression into a single scaffold did not yield increasingly higher mineralization as expected. This might be partly due to difficulty of the stain to access the interior of the multilayered compressed scaffolds (Figure 3).

3.4. Mechanical Testing. As expected, compression of 1layer scaffolds resulted in a decrease in sample thickness. For 2-layered scaffolds, thicknesses were higher for samples that were compressed first (C1M2) when compared to scaffolds that were compressed second (C2M1).
The opposite was observed in scaffolds with 4 layers in that scaffolds compressed second (C2M1) were thicker than those compressed first (C1M2). Scaffold thicknesses ranged between $130-315 \mu \mathrm{m}, 250-515 \mu \mathrm{m}$, and $700-1200 \mu \mathrm{m}$ for 1,2 , and 4 layers, respectively (Figure 4(a)). Compression of scaffolds before mineralization generally results in stiffer scaffolds. This finding is true for both PDO and PDO:nHA scaffolds. As expected, testing samples in dry state resulted in greater stiffness values compared to testing under hydrated conditions. However the effect of hydration is more obvious in PDO:nHA than the PDO scaffolds. Among the single layered scaffolds, non-compressed PDO:nHA was weaker than PDO but compression of PDO: nHA scaffolds resulted in scaffolds stiffer than compressed PDO scaffolds. In 2and 4-layered scaffolds, compression before mineralization resulted in stiffer scaffolds compared to scaffolds that were mineralized first. Further, as long as the PDO:nHA scaffolds were compressed before SBF treatment, increase in the 


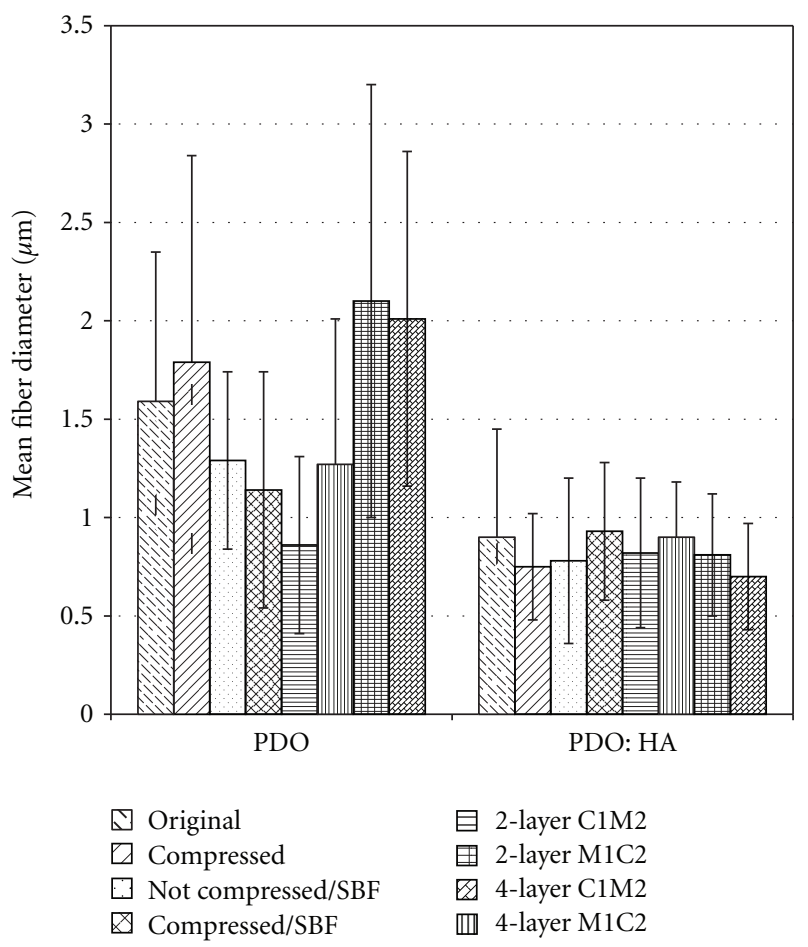

FIGURE 2: Fiber diameters of original, mineralized, and multilayered scaffolds.

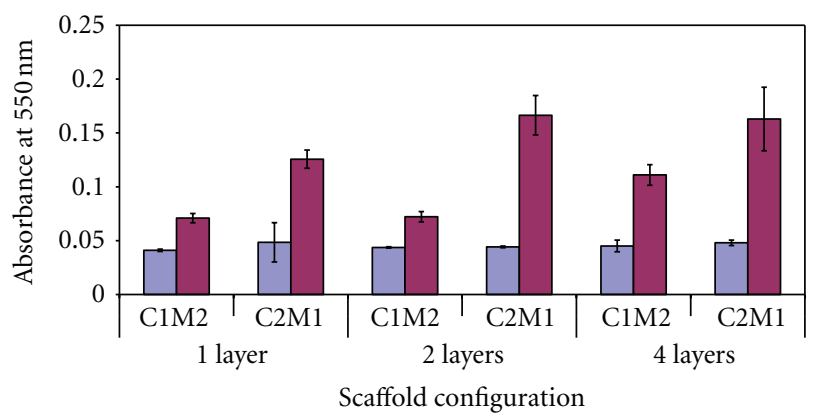

$\square$ PDO

$\square$ PDO: nHA

FIGURE 3: Alizarin red staining of multilayered scaffolds. Electrospun PDO and PDO:nHA scaffolds were stacked on top of each other to get 2 or 4 layers. One group was compressed first (C1M2) and mineralized as multilayered scaffold while the other group was mineralized as individual sheets and compressed into a single unit (C2M1) after SBF treatment. Following incubation, scaffolds were washed and stained for calcium using Alizarin red staining as described in Section 2.

number of layers resulted in proportionally greater stiffness values. However, the PDO scaffolds did not have any statistically significant difference whether it has 2 or 4 layers and are independent of compression before or after mineralization (Figure 4(b)).

For PDO:nHA scaffolds, strain at break was consistent (approximately 30\% and 50\% for dry and wet, resp.) independent of the number of layers and sequence of compression and mineralization. PDO scaffolds showed an increase in strain at break when mineralized first then compressed (C2M1) for both 2 and 4 layers (Figure 4(c)). For both PDO and PDO:nHA, energy to break was low (less than $2 \mathrm{~N}$. $\mathrm{mm}$ ) and unaltered when comparing 1-layer compressed and non-compressed scaffolds. For 2-layered scaffolds, energy to break increased slightly for PDO scaffolds compressed second (C2M1) and PDO:nHA scaffolds compressed first. The strongest of each scaffold type was 4-layered mineralized first (C2M1) with hydrated PDO having the highest energy to break (Figure $4(\mathrm{~d})$ ).

3.5. Permeability and Pore Size Measurements. The effects of compressing nonmineralized single or multilayered scaffold are presented in Figure 5. It can readily be seen that compression of PDO:nHA causes a significant reduction in permeability compared to PDO scaffolds (Figure 5). This finding corroborates with the SEM finding that showed distinct areas of fiber welding seen in all variations of PDO:nHA scaffolds. In contrast, PDO scaffolds have less densely packed fibers and are seemingly not affected by compression, at least on the surface. However, the permeability of single-layered PDO scaffolds paradoxically increases following compression. In general, compression of non-mineralized scaffolds results in decreased pore size of the scaffolds with the exception of single-layered PDO scaffolds that is unaffected by compression (Figure 6). While the compression of single and 2-layered PDO:nHA scaffolds resulted in similar reduction of pore size, the corresponding 4-layered scaffold was completely impermeable.

\section{Discussion}

Bone tissue scaffolds must be engineered to possess sufficient mechanical and biological properties. Inorganic hydroxyapatite, which forms almost $50 \%$ of healthy bone, is present in such high amounts to satisfy both the above criteria. It provides a brittle, incompressible phase that is effectively dispersed and closely associated with the tough collagenous organic phase. It is this interaction between phases having distinct material properties that contribute to unique mechanical properties of bone $[7,22]$.

SEM images revealed interesting insights into the scaffold behavior when compressed (at 20,000 psi for one minute) at the nanoscale. There are discrete areas of PDO:nHA scaffolds that have been pressure welded as a result of the compression process. Interestingly, such changes are obviously missing in pure PDO scaffolds. Since the PDO concentration was kept constant during electrospinning $(100 \mathrm{mg} / \mathrm{mL})$ for both types of scaffolds, this behavior teases out the important role of nHA (incorporated during electrospinning) in determining bulk material properties of the final scaffold.

The rationale of adding $\mathrm{nHA}$ in the present study was to provide nucleation sites for mineralization during subsequent treatment with SBF. It was never intended to contribute to the mechanical properties of the scaffold. In fact, the uncompressed PDO:nHA scaffold is considerably weaker than corresponding PDO scaffolds. However, the behavior 


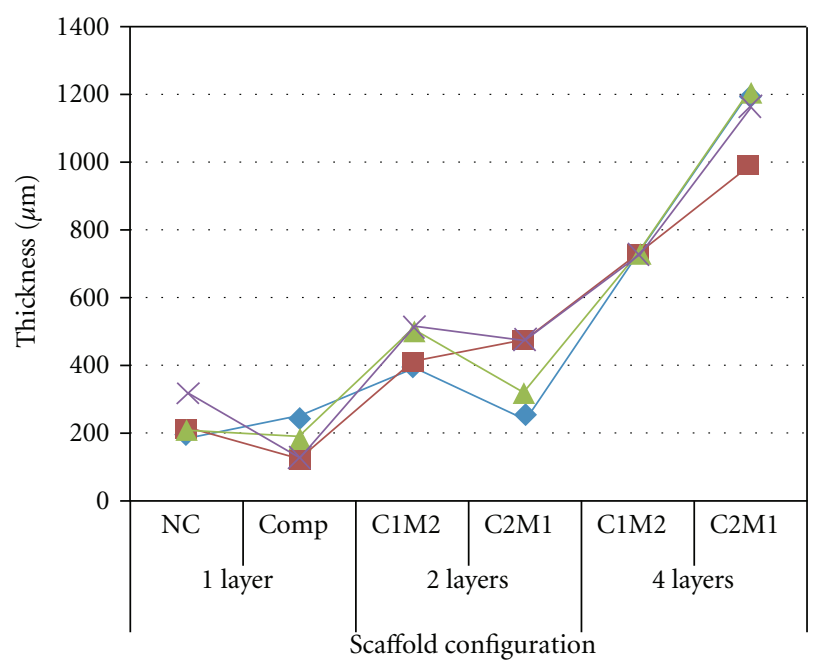

(a)

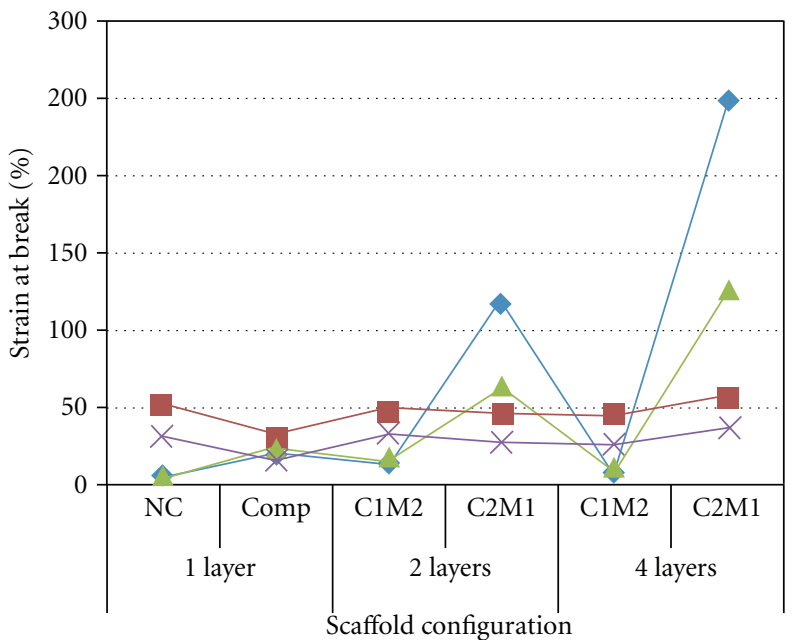

- Wet PDO
- Wet PDO: nHA

(c)

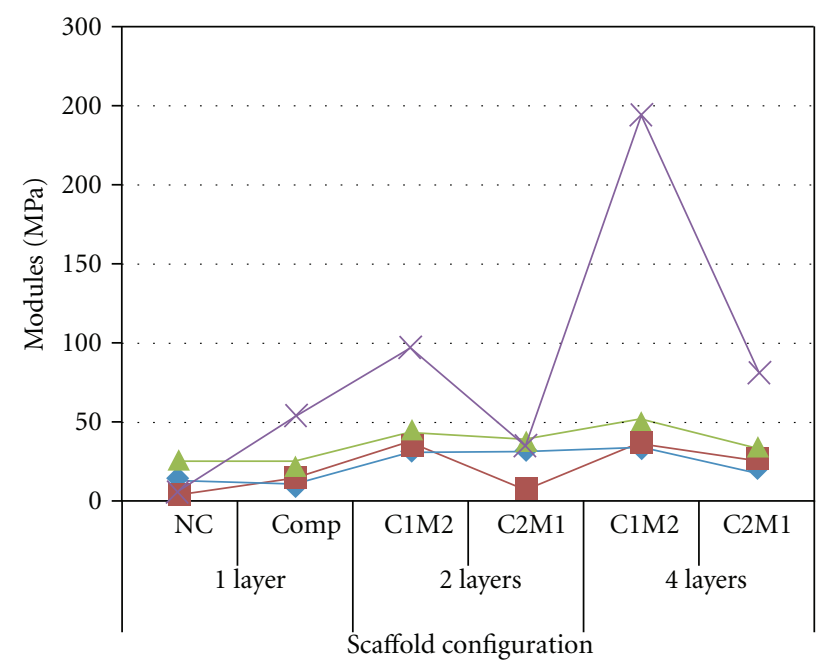

(b)

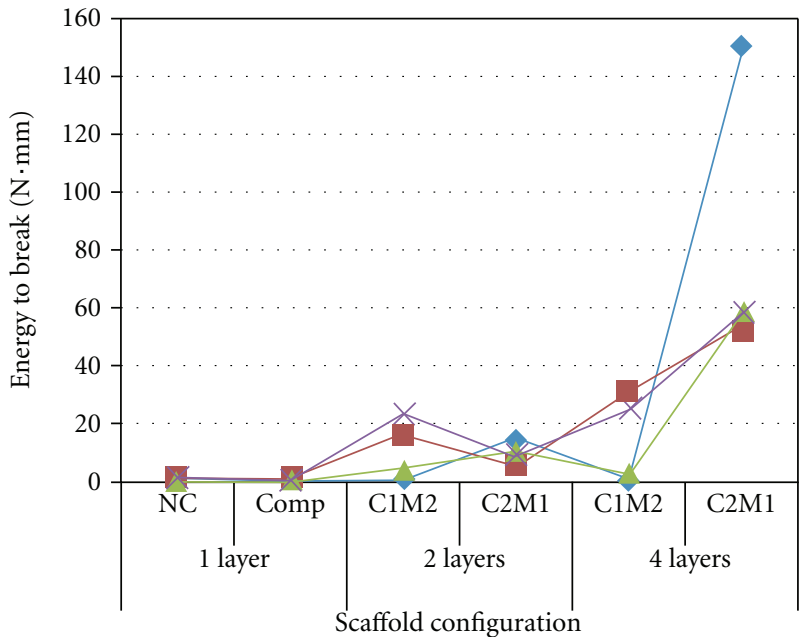

Wet PDO

Wet PDO: nHA

(d)

FiguRE 4: Comparison of (a) thickness, (b) stiffness (Young's modulus), (c) strain at break, and (d) energy to break in multilayered mineralized electrospun PDO and PDO:nHA scaffolds under dry and hydrated testing conditions. The effects of compression welding the scaffolds are also shown. In scaffolds containing more than 1-layer, compression welding of the scaffolds was done before SBF treatment (C1M2) or the scaffolds were mineralized separately and then compressed at the end of 2 weeks (C2M1). Non-compressed (NC), compressed (Comp).

of PDO:nHA scaffolds changes considerably under compression as shown in Figure 7. The stiffness of compressed PDO:nHA scaffolds shows a 7 -fold increase compared to non-compressed scaffolds. This observed increase in stiffness is further enhanced with the addition of layers, almost proportionally increasing with each layer. To date, these are amongst the highest reported modulus values for electrospun scaffolds. These findings provide a novel strategy to improve material properties of electrospun scaffolds. Even though the exact mechanism of improving stiffness is not clear at present, the following observations provide further insights into this interesting behavior.
Being more compressible, PDO:nHA scaffolds deform significantly under pressure that ultimately would lead to the increased density and welding of nanofibers. PDOs, on the other hand, being dense thin sheets, resist compression, even at the nanoscale. Further, an even compression of porous nanofibrous structure is limited by the occurrence of finite contact points of scaffolds with metal surfaces that are highly irregular at the nanoscale.

In addition to determining the mechanical properties, these nHA containing scaffolds are also expected to increase the amount of mineralization when soaked in SBF. However, 


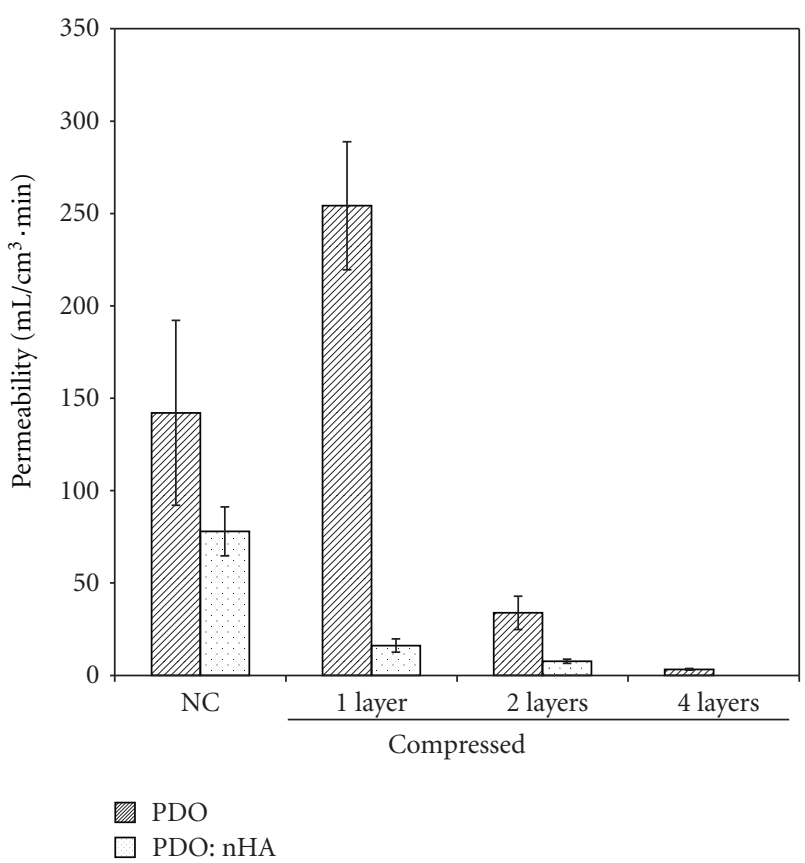

FIGURE 5: Effect on multilayered electrospun PDO and PDO:nHA scaffolds on permeability. Noncompressed (NC) scaffolds are single layers.

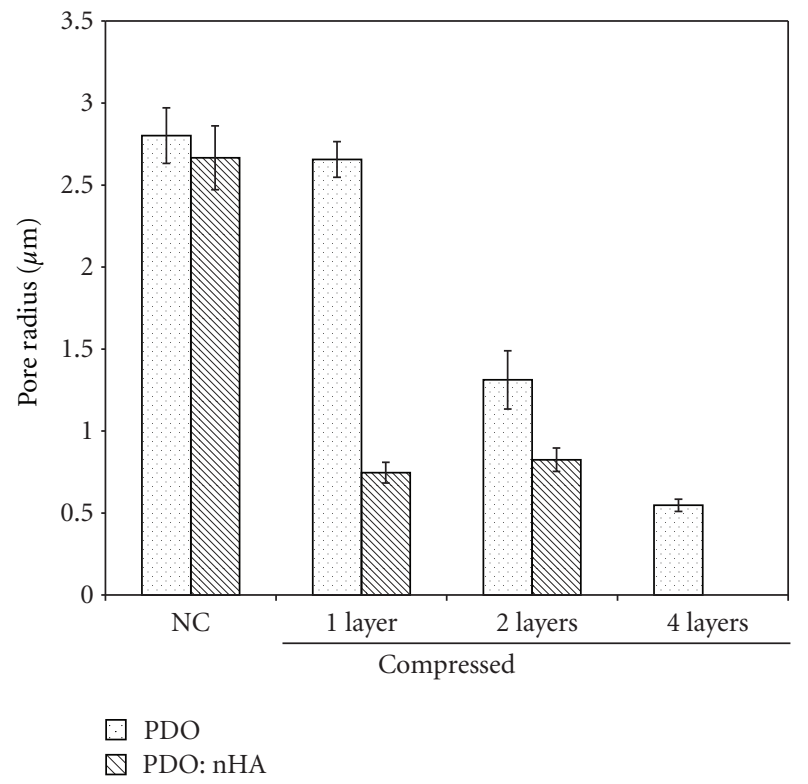

FIGURE 6: Effect of compression of PDO and PDO:nHA scaffolds on pore size. Non-compressed (NC) scaffolds are single layers.

mineralization of multiple scaffolds followed by their compression into a single scaffold did not yield greater mineralization outcome, at least in this study. This is most likely due to the limitation of staining process (alizarin red not able to bind to the mineral in the middle layers (Figure 3). A burnout test could be used to improve mineral quantification of multilayered scaffolds. Our lab has recently conducted

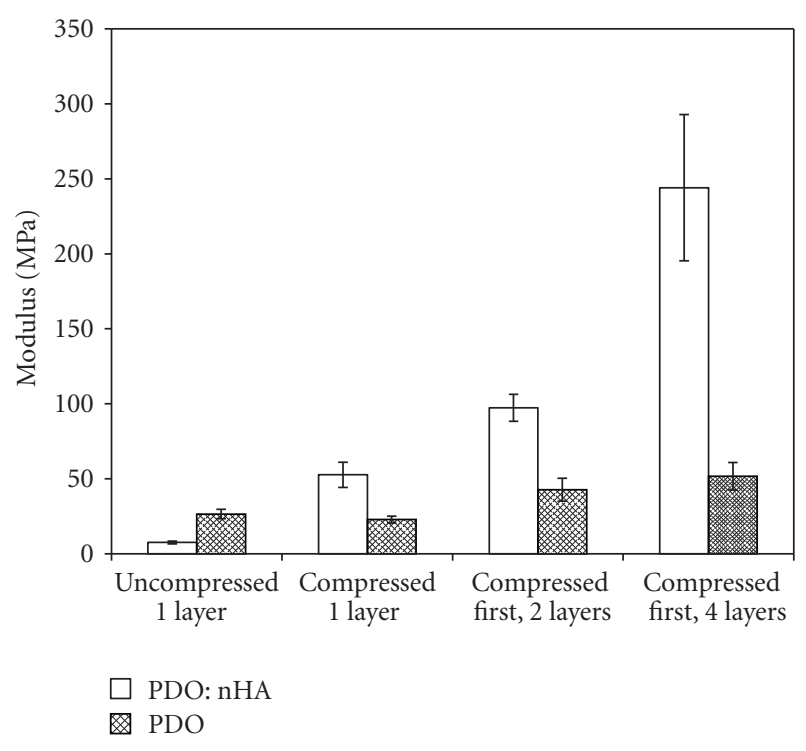

FIGURE 7: Effects of compression and multiple layers on the stiffness of electrospun PDO and PDO:nHA scaffolds.

preliminary studies using modified burn-out test specifically for mineralized scaffolds where samples are burned at $500^{\circ} \mathrm{C}$ until the polymer is burned off and only mineral ash remains [23]. This would be a more direct method of quantifying (by weight) the mineral content of multilayered scaffolds.

While the experiments unequivocally show a dramatic reduction in the porosity and permeability of electrospun PDO:nHA scaffolds following compression, the SEM still show fibrous morphology and exhibit pore structures at least on the surface. It is possible that the deeper layers are welded tightly resulting in decreased permeability. As tissue engineers, we expect the cells to first inhabit the surface and progressively migrate into the scaffolds. In this sense, we believe that the cells will perceive the compressed scaffold surface as favorable. Previous cell culture studies have confirmed the biocompatibility of electrospun PDO and PDO blends [24-26]. Since the tissue engineered scaffold is made up of biocompatible materials ( $\mathrm{PDO}$ and $\mathrm{nHA}$ ), we expect over time the polymeric scaffold will undergo enzymatic and non-enzymatic hydrolysis that will eventually permit cells to invade the depth of the scaffold.

Overall, the above experiments studied the effects of mineralization with SBF, and compression of multiple layers. Taken together these data represent a significant increase in the modulus following compression of multilayered scaffolds. But this process was accompanied with decreased mineralization and decreased permeability. There is essentially a middle ground between optimal mineralization and permeability vis-à-vis improved mechanical properties. Since mechanical properties of bone scaffolds are very critical in its function, further efforts should aim to identify methods to achieve significant mineralization and permeability without compromising mechanical properties. The use of air-flow impedance electrospinning is a viable fabrication technique in where 
the desired scaffold properties mentioned above could be achieved [27].

\section{Conclusion}

We have identified main processing parameters that affect two major properties of bone scaffolds: mechanical and mineral content. Use of high pressure (cold) welding of nanofibrous scaffolds greatly improves material properties while incubation of electrospun scaffolds in $1 \mathrm{x}$ r-SBF provides a convenient and physiological means to improve mineralization of scaffolds in vitro. Compression of electrospun scaffolds at very high pressure resulted in significantly higher levels of stiffness of PDO:nHA scaffolds compared to uncompressed PDO and PDO:nHA controls. The recorded modulus values for layered PDO:nHA scaffolds are amongst the highest reported for electrospun scaffolds. In addition, this technique also permitted stacking of multiple independent electrospun scaffolds on top of each other without fear of delamination. On the other hand, such an approach limits the amount of mineralization following treatment with SBF due to loss of available surface area. A 4-layered PDO:nHA scaffold compressed first followed by mineralization in $\mathrm{r}$-SBF has maximal stiffness with the mineralization second only to non-compressed scaffolds. Within the limitation of this study, this scaffold configuration represents an optimal midway for desired stiffness and mineral content.

\section{References}

[1] J. O. Hollinger and A. Chaudhari, "Bone regeneration materials for the mandibular and craniofacial complex," Cells and Materials, vol. 4, pp. 143-151, 1992.

[2] M. V. Hillsley and J. A. Frangos, "Bone tissue engineering: the role of interstitial fluid flow," Biotechnology and Bioengineering, vol. 43, no. 7, pp. 573-581, 1994.

[3] D. W. Hutmacher, "Scaffolds in tissue engineering bone and cartilage," Biomaterials, vol. 21, no. 24, pp. 2529-2543, 2000.

[4] J. Y. Rho, L. Kuhn-Spearing, and P. Zioupos, "Mechanical properties and the hierarchical structure of bone," Medical Engineering and Physics, vol. 20, no. 2, pp. 92-102, 1998.

[5] B. D. Ratner, A. S. Hoffman, F. J. Schoen et al., "Orthopedic materials. Application of materials in medicine, biology and artificial organs," in Biomaterials Science: An Introduction to Materials in Medicine, Elsevier-Academic Press, Calif, USA, 2nd edition, 2004.

[6] D. W. Hutmacher, J. T. Schantz, C. X. Lam, K. C. Tan, and T. C. Lim, "State of the art and future directions of scaffold-based bone engineering from a biomaterials perspective," Journal of Tissue Engineering and Regenerative Medicine, vol. 1, no. 4, pp. 245-260, 2007.

[7] J. D. Currey, "The design of mineralised hard tissues for their mechanical functions," Journal of Experimental Biology, vol. 202, no. 23, pp. 3285-3294, 1999.

[8] P. Fratzl, H. S. Gupta, E. P. Paschalis, and P. Roschger, "Structure and mechanical quality of the collagen-mineral nanocomposite in bone," Journal of Materials Chemistry, vol. 14, no. 14, pp. 2115-2123, 2004.
[9] S. J. Peter, M. J. Miller, A. W. Yasko, M. J. Yaszemski, and A. G. Mikos, "Polymer concepts in tissue engineering," Journal of Biomedical Materials Research, vol. 43, no. 4, pp. 422-427, 1998.

[10] J. H. Jang, O. Castano, and H. W. Kim, "Electrospun materials as potential platforms for bone tissue engineering," Advanced Drug Delivery Reviews, vol. 61, no. 12, pp. 1065-1083, 2009.

[11] C. P. Barnes, S. A. Sell, E. D. Boland, D. G. Simpson, and G. L. Bowlin, "Nanofiber technology: designing the next generation of tissue engineering scaffolds," Advanced Drug Delivery Reviews, vol. 59, no. 14, pp. 1413-1433, 2007.

[12] S. A. Sell, M. J. McClure, K. Garg, P. S. Wolfe, and G. L. Bowlin, "Electrospinning of collagen/biopolymers for regenerative medicine and cardiovascular tissue engineering," Advanced Drug Delivery Reviews, vol. 61, no. 12, pp. 1007-1019, 2009.

[13] S. S. Liao, F. Z. Cui, W. Zhang, and Q. L. Feng, "Hierarchically biomimetic bone scaffold materials: nano-HA/collagen/PLA composite," Journal of Biomedical Materials Research Part B, vol. 69, no. 2, pp. 158-165, 2004.

[14] M. P. Prabhakaran, J. Venugopal, and S. Ramakrishna, "Electrospun nanostructured scaffolds for bone tissue engineering," Acta Biomaterialia, vol. 5, no. 8, pp. 2884-2893, 2009.

[15] J. Venugopal, M. P. Prabhakaran, Y. Zhang, S. Low, A. T. Choon, and S. Ramakrishna, "Biomimetic hydroxyapatitecontaining composite nanofibrous substrates for bone tissue engineering," Philosophical Transactions of the Royal Society A, vol. 368, no. 1917, pp. 2065-2081, 2010.

[16] D. Gupta, J. Venugopal, S. Mitra, V. R. Giri Dev, and S. Ramakrishna, "Nanostructured biocomposite substrates by electrospinning and electrospraying for the mineralization of osteoblasts," Biomaterials, vol. 30, no. 11, pp. 2085-2094, 2009.

[17] R. Zhang and P. X. Ma, "Porous poly(L-lactic acid)/apatite composites created by biomimetic process," Journal of Biomedical Materials Research, vol. 45, no. 4, pp. 285-293, 1999.

[18] A. Oyane, H. M. Kim, T. Furuya, T. Kokubo, T. Miyazaki, and T. Nakamura, "Preparation and assessment of revised simulated body fluids," Journal of Biomedical Materials Research Part A, vol. 65, no. 2, pp. 188-195, 2003.

[19] P. A. Madurantakam, I. A. Rodriguez, C. P. Cost et al., "Multiple factor interactions in biomimetic mineralization of electrospun scaffolds," Biomaterials, vol. 30, no. 29, pp. 5456-5464, 2009.

[20] C. A. Gregory, W. G. Gunn, A. Peister, and D. J. Prockop, "An Alizarin red-based assay of mineralization by adherent cells in culture: comparison with cetylpyridinium chloride extraction," Analytical Biochemistry, vol. 329, no. 1, pp. 77-84, 2004.

[21] S. Sell, C. Barnes, D. Simpson, and G. Bowlin, "Scaffold permeability as a means to determine fiber diameter and pore size of electrospun fibrinogen," Journal of Biomedical Materials Research Part A, vol. 85, no. 1, pp. 115-126, 2008.

[22] K. L. Kilpadi, P. L. Chang, and S. L. Bellis, "Hydroxylapatite binds more serum proteins, purified integrins, and osteoblast precursor cells than titanium or steel," Journal of Biomedical Materials Research, vol. 57, no. 2, pp. 258-267, 2001.

[23] I. A. Rodriguez, P. A. Madurantakam, J. M. McCool et al., "Mineralization potential of electrospun PDO-hydroxyapatitefibrinogen blended scaffolds," International Journal of Biomaterials, vol. 2012, Article ID 159484, 12 pages, 2012.

[24] S. A. Sell, M. J. McClure, C. P. Barnes et al., "Electrospun polydioxanone-elastin blends: potential for bioresorbable vascular grafts," Biomedical Materials, vol. 1, no. 2, pp. 72-80, 2006.

[25] W. N. Chow, D. G. Simpson, J. W. Bigbee, and R. J. Colello, "Evaluating neuronal and glial growth on electrospun polarized 
matrices: bridging the gap in percussive spinal cord injuries," Neuron Glia Biology, vol. 3, no. 2, pp. 119-126, 2007.

[26] M. P. Francis, P. C. Sachs, P. A. Madurantakam et al., "Electrospinning adipose tissue-derived extracellular matrix for adipose stem cell culture," Journal of Biomedical Materials Research Part A, vol. 100, no. 7, pp. 1716-1724, 2012.

[27] M. J. McClure, P. S. Wolfe, D. G. Simpson, S. A. Sell, and G. L. Bowlin, "The use of air-flow impedance to control fiber deposition patterns during electrospinning," Biomaterials, vol. 33 , no. 3, 7719 pages, 2012 . 

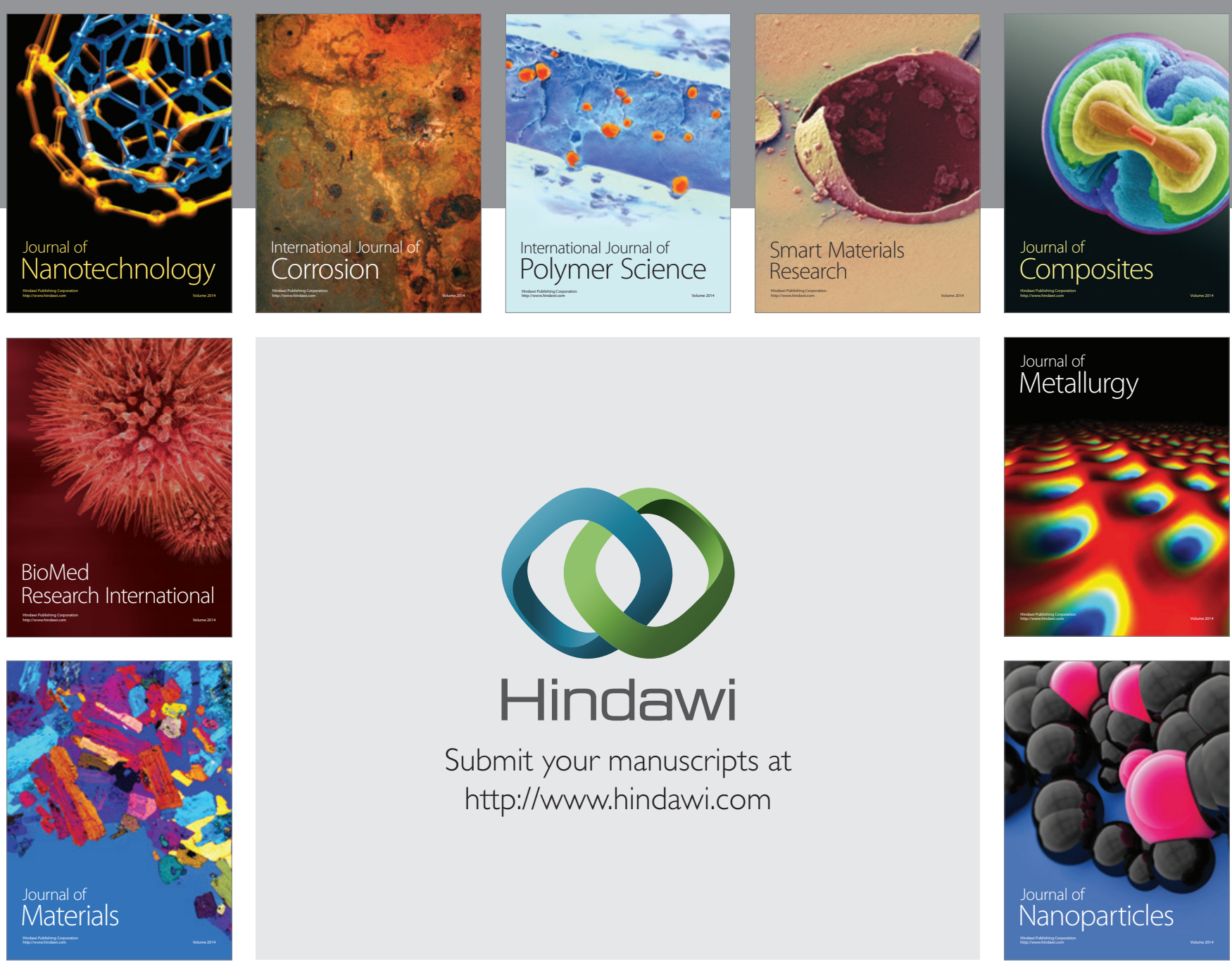

Submit your manuscripts at http://www.hindawi.com
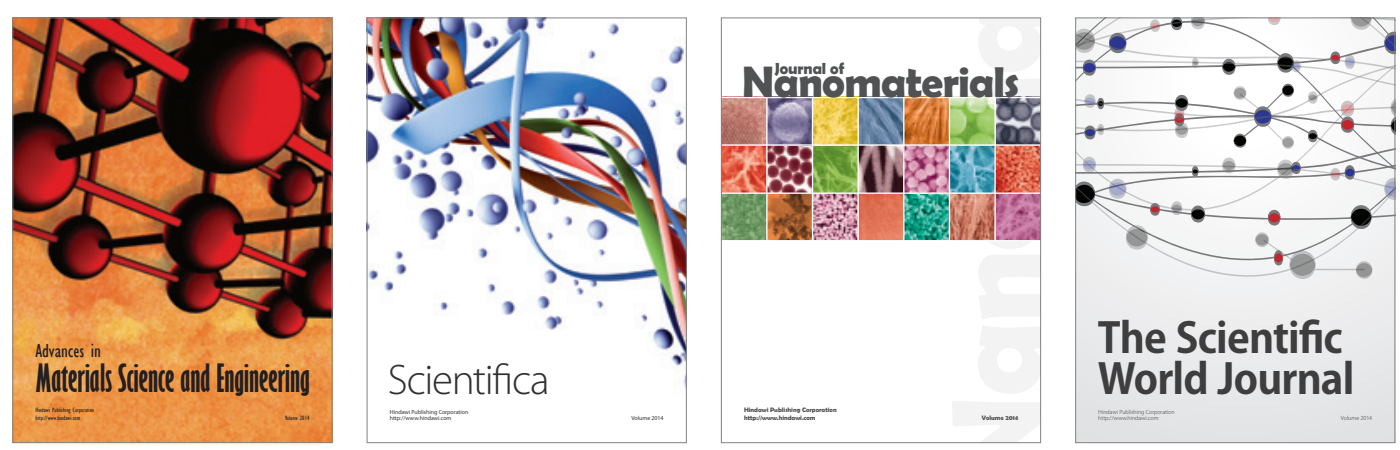

\section{The Scientific World Journal}
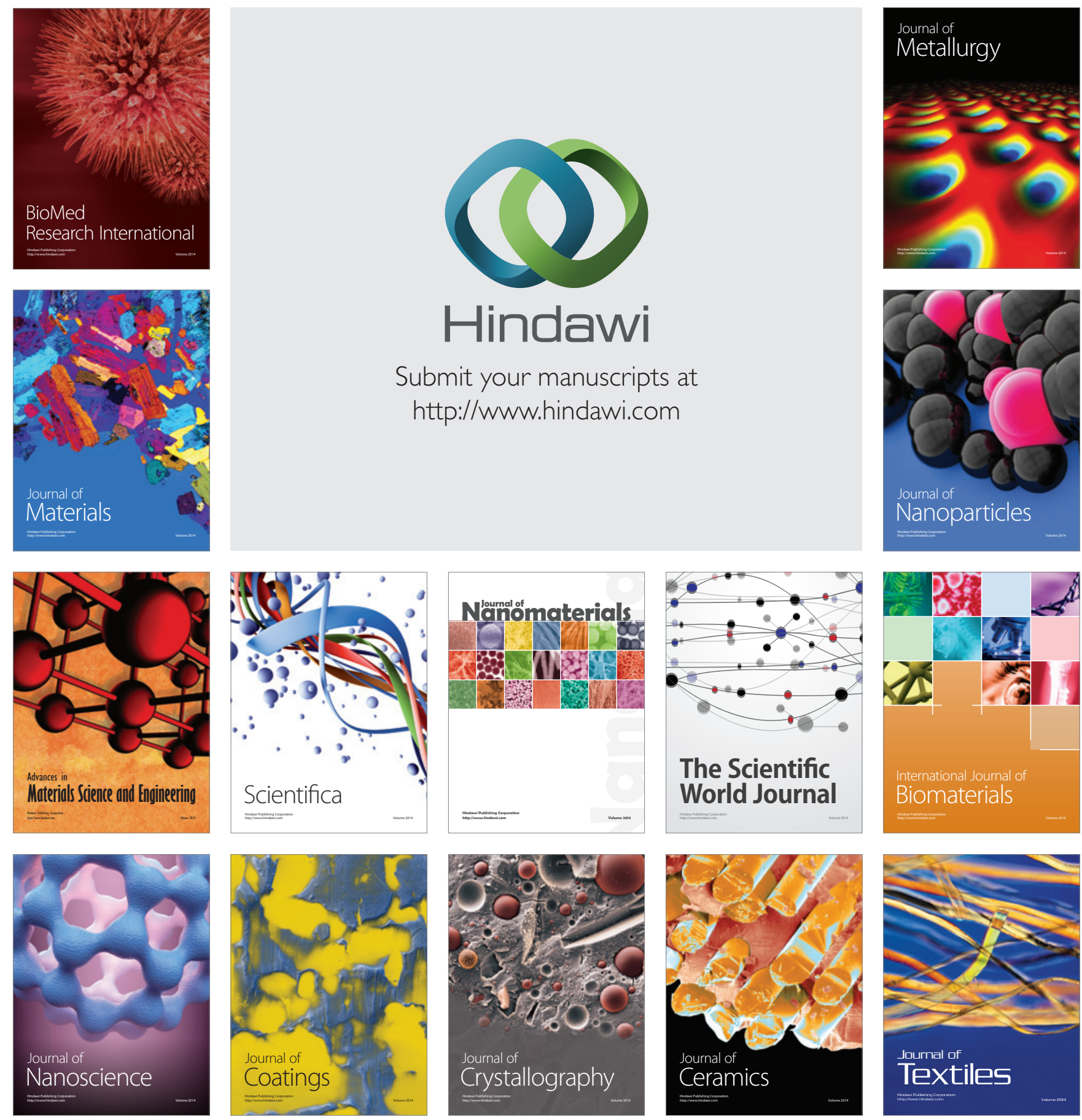